\title{
Publicações sobre Ensino em Enfermagem na Revista da Escola de Enfermagem da USP
}

\author{
PUBLICATIONS ABOUT NURSING EDUCATION INTHE \\ REVISTADA ESCOLADE ENFERMAGEM DA USP
}

\author{
PUBLICACIONES SOBRE ENSEÑANZA EN ENFERMERÍA \\ EN LA REVISTA DA ESCOLA DE ENFERMAGEM DA USP
}

\author{
Elaine Emi Ito', Regina Toshie Takahashi²
}

\begin{abstract}
RESUMO
Produções científicas na área de ensino em enfermagem fornecem importantes contribuições para a formação de profissionais e exercício da enfermagem. Acreditando nesta perspectiva, foi realizado um estudo visando a identificar e analisar os artigos publicados na área de ensino em enfermagem, construindo categorias empíricas com base nos dados coletados. Trata-se de uma pesquisa bibliográfica, descritiva e quantitativa, tendo como fonte artigos publicados na Revista da Escola de Enfermagem da USP, durante o período de 1967 a 2002. Foram encontrados 1.137 artigos, sendo que $14,2 \%$ destes referem-se ao ensino de enfermagem, dos quais $89,0 \%$ dizem respeito à área de graduação. A análise descritiva dos artigos publicados, referentes ao ensino de enfermagem, desperta a preocupação sobre o desenvolvimento de pesquisas relacionadas ao tema e que acompanhem as mudanças das legislações e as exigências do mercado de trabalho.
\end{abstract}

\section{DESCRITORES}

Educação em enfermagem.

Pesquisa em educação de

Enfermagem.

Publicações periódicas.

\begin{abstract}
Scientific production in nursing education field provide important contributions to professional formation and nursing practice. Since we believe this perspective, we carried out a survey aimed at identifying and analyzing nursing education-related articles, building empiric categories from the collected data. This revision is a bibliographical, descriptive and quantitative survey using as source articles published in the Revista da Escola de Enfermagem da USP from 1967 to 2002 . We have found 1,137 articles, of which $14.2 \%$ refer to nursing education, $89.0 \%$ of which are concerned with the undergraduate level. The descriptive analysis of nursing education-related bibliography gives rise to issues concerning the development of research that follow changes in the legislation changes and the demands of the professional market.
\end{abstract}

\section{KEY WORDS}

Education, nursing.

Nursing education research.

Periodicals.

\section{RESUMEN}

Producciones científicas en el área de la enseñanza en enfermería ofrecen importantes contribuciones para la formación de profesionales y el ejercicio de la enfermería. Creyendo en esta perspectiva, fue realizado un estudio visando identificar y analizar los artículos publicados en el área de la enseñanza en enfermería, construyendo categorías empíricas a partir de los datos recolectados. Se trata de una investigación bibliográfica, descriptiva y cuantitativa, teniendo como fuente artículos publicados en la Revista da Escola de Enfermagem da USP, durante el período de 1967 a 2002. Fueron encontrados 1,137 artículos de los cuales el $14,2 \%$ se refieren a la enseñanza de enfermería, y de éstos el 89,0\% se refieren al área del pre grado. El análisis descriptivo de los artículos publicados, referentes a la enseñanza de enfermería, despierta preocupación sobre el desarrollo de investigaciones relacionadas al tema y que acompañen los cambios de las legislaciones y las exigencias del mercado de trabajo.

\section{DESCRIPTORES}

Educación en enfermería. Investigación en educación de enfermería.

Publicaciones periódicas.
1 Enfermeira do Serviço de Educação Continuada do Hospital Santa Marcelina, Especialista em Administração de Serviços de Saúde, Mestranda pela Escola de Enfermagem da USP (EEUSP). emi.ito@uol.com.br

2 Enfermeira, Professora Doutora do Departamento de Orientação Profissional da EEUSP. 
Elaine Emi lto

Regina Toshie Takahashi

\section{INTRODUÇÃO}

O ensino tem a necessidade de mudanças constantes de acordo com as exigências encontradas em cada época. As mudanças em nossa sociedade e em nossas políticas de saúde são fatores determinantes para a construção do ensino de enfermagem e formação de profissionais engajados na realidade ${ }^{(1)}$.

No Brasil, o ensino de enfermagem passou por várias etapas de desenvolvimento ao longo dos anos, refletindo, em cada mudança, parte do contexto histórico da enfermagem e da sociedade brasileira.

Em 1890, houve a primeira evidência de organização do ensino de enfermagem no Brasil, com a criação de uma escola de enfermagem no Rio de Janeiro denominada Escola Alfredo Pinto, que nasce no Hospital Nacional de Alienados, com objetivos dirigidos à formação de pessoal para atuar em psiquiatria, hospitais civis e militares ${ }^{(2)}$.

Embora a Escola Alfredo Pinto tenha sido considerada a primeira Escola de Enfermagem do Brasil, uma escola com organização administrativa e docente, sob a responsabilidade de enfermeiros, surge somente em 1923, no Rio de Janeiro, com a Escola Ana Néri.

Nessa época, começa a emergir o ensino sistematizado da enfermagem, com o propósito de formar profissionais que contribuíssem para garantir o saneamento dos portos, em virtude do agravamento do quadro de saúde pública no Brasil, que refletiu nas relações comerciais.

O currículo de enfermagem implantado em 1923 ficou inalterado até 1949 , ano em que sofreu a primeira reformulação e passou a ser regulado pela Lei $\mathrm{n}^{\circ} 775 / 49$, regulamentado pelo Decreto $\mathrm{n}^{\circ} 27426 / 49$ que contribuiu efetivamente para a evolução da enfermagem no Brasil. Esta Lei fixou requisitos mínimos equivalentes ao ensino de enfermagem, tais como: exigência do ensino médio completo dos candidatos, existência de um currículo uniforme, estipulando a duração do curso em quatro anos. Manteve os princípios de que o diretor da escola de enfermagem deveria ser enfermeiro e que as disciplinas específicas de enfermagem deveriam ser ministradas por enfermeiros $^{(3)}$. Esta mesma lei estabeleceu também condições mínimas para a preparação de auxiliares de enfermagem, que na época era um curso de 18 meses essencialmente profissionalizante e dirigido para a assistência curativa, sendo exigido dos candidatos o certificado de conclusão do curso primário ${ }^{(4)}$.

Em 1961, com a Lei n ${ }^{\circ} 2604 / 55$, regulamentada pelo Decreto $\mathrm{n}^{\circ} 50387 / 61$, sobre o exercício da en- fermagem, deu-se ao enfermeiro o poder de mando em relação aos auxiliares, atendentes de enfermagem e aos cuidados de enfermagem ${ }^{(5)}$.

Com o parecer do Conselho Federal de Educação CFE n. ${ }^{\circ}$ 271/62, em 1962, o currículo mínimo do curso de enfermagem sofreu algumas modificações em relação à duração, sendo reduzida para 36 meses, e as disciplinas enfocavam um caráter curativo, modelo capitalista e centrado nas clínicas especializadas $^{(6)}$.

Este último currículo não atendeu às reivindicações pretendidas pelos enfermeiros, entidades de classe e escolas de enfermagem, fato que contribuiu para mudanças curriculares tendo como fator determinante a Reforma Universitária que criou a oportunidade de modificar esse currículo mínimo.

Com a Reforma Universitária de 1968, iniciam-se esforços para a capacitação de docentes, intensificam-se os cursos de especialização e criam-se cursos de mestrado. Observa-se que os cursos de pósgraduação desta época preparavam os docentes e pesquisadores para especialidades voltadas para a área hospitalar, visto que o currículo mínimo fixado priorizava a assistência curativa, considerando os hospitais como centros hegemônicos da assistência à saúde ${ }^{(4)}$.

Um novo currículo foi aprovado em 1972 pelo Parecer 163/72 e Resolução 4/72 do CFE. Porém, após discussões acerca da necessidade de reformulação do currículo, realizadas na década de 80 , no que se refere à formação profissional, conclui-se que o mesmo não seria mais capaz de atender as necessidades impostas pela área da saúde do Brasil, sendo iniciado novo debate para mudanças curriculares, concretizadas com a reformulação curricular de $1994^{(7)}$.

O novo currículo, respaldado no Parecer 314/94 e na Portaria 1721/94, previu a formação do enfermeiro em quatro áreas: assistência, gerência, ensino e pesquisa. Teve como pressuposto a educação como possibilidade de transformação, centrada na formação da consciência crítica, levando o enfermeiro à reflexão sobre a prática profissional e ao compromisso com a sociedade.

Quanto a formação do Técnico de Enfermagem, o primeiro curso foi criado em 1966 nas Escolas Ana Néri e Luíza de Marillac, pelos Pareceres do CFE ${ }^{\circ}$ 171/66 e $\mathrm{n}^{\circ} 5692 / 71$, quando passaram a integrar o Sistema Educacional Brasileiro ${ }^{(4)}$.

O Auxiliar de enfermagem passou a ser aquele que, após ter cumprido a escolaridade de ensino fundamental, recebia uma habilitação profissiona- 
lizante. Ao nível de ensino médio era enquadrado na categoria de Técnico de Enfermagem. A diferença entre o auxiliar e o técnico de enfermagem faz-se em função da carga horária tanto teórica como prática do curso freqüentado(4).

Ainda neste contexto histórico do ensino da enfermagem no Brasil é importante ressaltar que, em virtude da nova Lei de Diretrizes e Bases da Educação Nacional (LDB)- Lei n 9394 de 20/12/96 há inovações e mudanças na educação nacional assegurando às instituições de ensino superior autonomia didático-científica, bem como, autonomia em fixar os currículos dos seus cursos e programas. Assim, as universidades não têm a obrigatoriedade em seguir a regulamentação do currículo mínimo determinada pela Portaria 1721/94. No momento atual, o currículo não é mais o único determinante, mas base para direcionar e orientar o ensino de graduação em enfermagem $^{(8)}$.

A nova LDB trouxe novas responsabilidades para as instituições de ensino superior, docentes, discentes e sociedade, pois permite a formação de diferentes perfis profissionais a partir da vocação de cada curso/ escola, esperando melhor adaptação ao mercado de trabalho, já que as instituições terão a liberdade para definir parte considerável de seus currículos plenos $^{(9)}$.

A Resolução 3/2001 do Conselho Nacional de Educação ${ }^{(10)}$ determina em seu Artigo $3^{\circ}$ do Curso de Graduação em Enfermagem que o perfil do formando egresso/profissional seja:

I - Enfermeiro, com formação generalista, humanista, crítica e reflexiva. Profissional qualificado para o exercício de enfermagem, com base no rigor científico e intelectual e pautado em princípios éticos. Capaz de conhecer e intervir sobre os problemas / situações de saúde doenças mais prevalentes no perfil epidemiológico nacional, com ênfase na sua região de atuação, identificando as dimensões bio-psico-sociais dos seus determinantes. Capacitado a atuar, com senso de responsabilidade e compromisso com a cidadania, como promotor da saúde integral do ser humano.

Acrescenta, ainda, em seu Artigo $4^{\circ}$ que a formação do enfermeiro tem por objetivo dotar o profissional de conhecimentos requeridos para o exercício das seguintes competências e habilidades gerais: Atenção à saúde; Tomada de decisões; Comunicação; Liderança; Administração e Gerenciamento; Educação permanente ${ }^{(10)}$.

Apesar do Conselho Nacional de Educação ${ }^{(10)}$ não definir as disciplinas básicas que deverão conter os currículos dos Cursos de Graduação em En- fermagem, define princípios, fundamentos, condições e procedimentos que direcionam a formação do profissional Enfermeiro.

No mercado de trabalho, atualmente, encontramos três profissionais na área de enfermagem (enfermeiros, técnicos e auxiliares de enfermagem), que possuem diferentes níveis de conhecimentos e habilidades. Entretanto, estas categorias profissionais têm o mesmo objeto de trabalho: o cuidado ao ser humano. Portanto, a preocupação com a formação destes profissionais deve ser constante.

O processo ensino-aprendizagem na enfermagem, em todos os níveis de ensino, possui certas peculiaridades por ser eminentemente teórico-prático e sua aplicação ocorrer no ser humano, o qual possui direitos, conhecimento ético e político, sentimentos e necessidades distintas.

O contato do aluno com a realidade da enfermagem em campo permite que este conheça e identifique as atividades que permeiam a prática assistencial e a maneira de ser e fazer enfermagem. Portanto, as instituições de ensino e as instituições prestadoras de serviços de saúde, campo da prática, devem estabelecer vínculos, proporcionando ao aluno o aprendizado da enfermagem, no contexto da prática para a formação de profissionais qualificados.

A responsabilidade da qualidade de formação dos enfermeiros, técnicos e auxiliares de enfermagem, não é só dos órgãos formadores, é também das instituições prestadoras de assistência à saúde, possibilitando evitar a dicotomia entre o que é ensinado e o que é encontrado na prática ${ }^{(11)}$.

Um problema que usualmente aparece em estágio, é a dificuldade que os alunos possuem em realizarem a transição do ensino da sala de aula ao ensino clínico, e do conhecimento adquirido na teoria ser aplicado em situações práticas. O entrosamento entre a instituição de ensino, instituição de saúde e os alunos é um vital componente para o aprendizado em estágio ser eficiente, alcançando os objetivos traçados pela metodologia de ensino ${ }^{(12)}$.

Como enfermeira do Serviço de Educação Continuada de um hospital considerado Instituição de Ensino, que recebe alunos de nível médio, técnico e de graduação em enfermagem para estágios, tenho o interesse em verificar e analisar os artigos publicados sobre o ensino de enfermagem, acreditando na perspectiva da importância de enfermeiros ligados ao campo da prática identificarem o direcionamento das publicações realizadas nesta área e, assim, contribuir no processo ensino aprendizagem dos discentes no âmbito da prática.
Publicações sobre Ensino em Enfermagem na Revista da Escola de Enfermagem da USP 
Elaine Emi lto

Regina Toshie Takahashi
Desta forma, foi realizado um estudo sobre as publicações na área de ensino em enfermagem na Revista da Escola de Enfermagem da Universidade de São Paulo (REEUSP), criada em 1967, e que tem sido, ao longo dos anos um dos principais meios de divulgação dos conhecimentos na área de enfermagem.

\section{OBJETIVO}

Verificar e analisar os artigos publicados sobre o ensino de enfermagem quanto ao tipo de artigo, de acordo com a categorização da REEUSP, o autor, o ano, o nível de ensino que se refere o artigo e a temática estudada.

\section{MATERIAL E MÉTODO}

$\mathrm{O}$ presente estudo tem enfoque de pesquisa bibliográfica, descritiva e quantitativa, tendo como fonte os artigos publicados na Revista da Escola de Enfermagem da Universidade de São Paulo (REEUSP), desde 1967 à 2002, com o propósito de verificar os aspectos abordados nos artigos sobre o ensino de enfermagem nos níveis médio, graduação e pós graduação.

A pesquisa bibliográfica procura explicar um problema a partir de referências teóricas publicadas em documentos, busca conhecer e analisar as contribuições culturais ou científicas do passado existente sobre um determinado assunto, tema ou problema. E, quando esta constitui parte da pesquisa descritiva, é feita com o intuito de recolher informações e conhecimentos prévios acerca de um problema para o qual se procura uma resposta ou uma hipótese que se quer experimentar ${ }^{(13)}$.

O presente estudo foi realizado nas publicações encontradas na Revista da Escola de Enfermagem da Universidade de São Paulo, criada em 1967, inicialmente semestral, em 1975 passou a ser publicada quadrimestralmente e em 1998 até hoje, é editada com uma periodicidade trimestral, nos meses de março, junho, setembro e dezembro, tendo eventualmente a publicação de números especiais.

A Revista é editada e publicada no município de São Paulo e tem como finalidades: 1. apresentar bibliografia necessária ao ensino e ao exercício da enfermagem; 2 . oferecer aos docentes um veículo para publicação de seus trabalhos. O público a que se destina é principalmente para enfermeiros e estudantes de enfermagem ${ }^{(14)}$.

Atualmente, a revista é indexada em bases de dados nacionais e internacionais (CINAHL,
MEDLINE e LILACS) e também em obras de referência impressas (International Nursing Index, Bibliografia Brasileira de Enfermagem, Periódica: Índice de Revista Latino-Americana em Ciências e Banco de Dados em Enfermagem).

Foi realizado, pelas pesquisadoras, o levantamento de todas as revistas publicadas desde o seu início em 1967 até 2002, verificando no sumário os temas referentes ao ensino de enfermagem. Após a identificação do tema, foi realizada leitura dos resumos, palavras chave e rodapés de cada artigo encontrado. Estes foram classificados, agrupados e sistematizados nos seguintes aspectos:

- Categoria dos artigos: foram classificados, de acordo com as categorias especificadas pela REEUSP: pesquisa, revisão crítica da literatura, nota e discussão, relato de experiência profissional e resumo de teses ou dissertações.

- Autor: foram classificados pela ocupação principal, sendo estes: docente de enfermagem, aluno de graduação, aluno de pós graduação, enfermeiro assistencial e sem classificação aos que não haviam especificação da ocupação do autor.

- Nível de ensino a que se refere o artigo: foram considerados os níveis de ensino médio, graduação e pós graduação.

- Temática do artigo: foram identificados 08 ítens no contexto do ensino de enfermagem - avaliação, estratégia de ensino, docente, aluno, vestibular, estágio, currículo e conteúdo de disciplinas.

- A frequiência das categorias dos artigos foram distribuídas em períodos de 4 em 4 anos para facilitar a apresentação dos gráficos.

Os resultados e sua apresentação foram demonstrados em tabelas e gráficos e sua análise foi baseada em números percentuais e absolutos.

\section{RESULTADOS}

No estudo foram encontradas 89 revistas publicadas ininterruptamente desde setembro de 1967 até dezembro de 2002, e verificados 1137 artigos publicados. Destes, foram identificados 161 $(14,2 \%)$ artigos referentes ao ensino de enfermagem no Brasil, considerando o nível médio, de graduação e de pós-graduação.

A quantidade de publicações sobre o ensino de enfermagem variou ao longo dos 36 anos pesquisados. O período foi dividido de quatro em quatro anos para facilitar a apresentação em gráfico. 
Podemos observar, no Gráfico 1, a freqüência e a porcentagem dos artigos publicados sobre o tema em questão. Na década de 90 verifica-se um aumento importante no número de artigos. Nesta época, acentuavam-se as discussões e revisões do currículo do curso de graduação das escolas de enfer- magem, subsidiando a proposta de reformulação do currículo mínimo vigente na época, reforçando a necessidade da formação de enfermeiros críticos e reflexivos estimulados no desenvolvimento do espírito científico ${ }^{(9)}$.

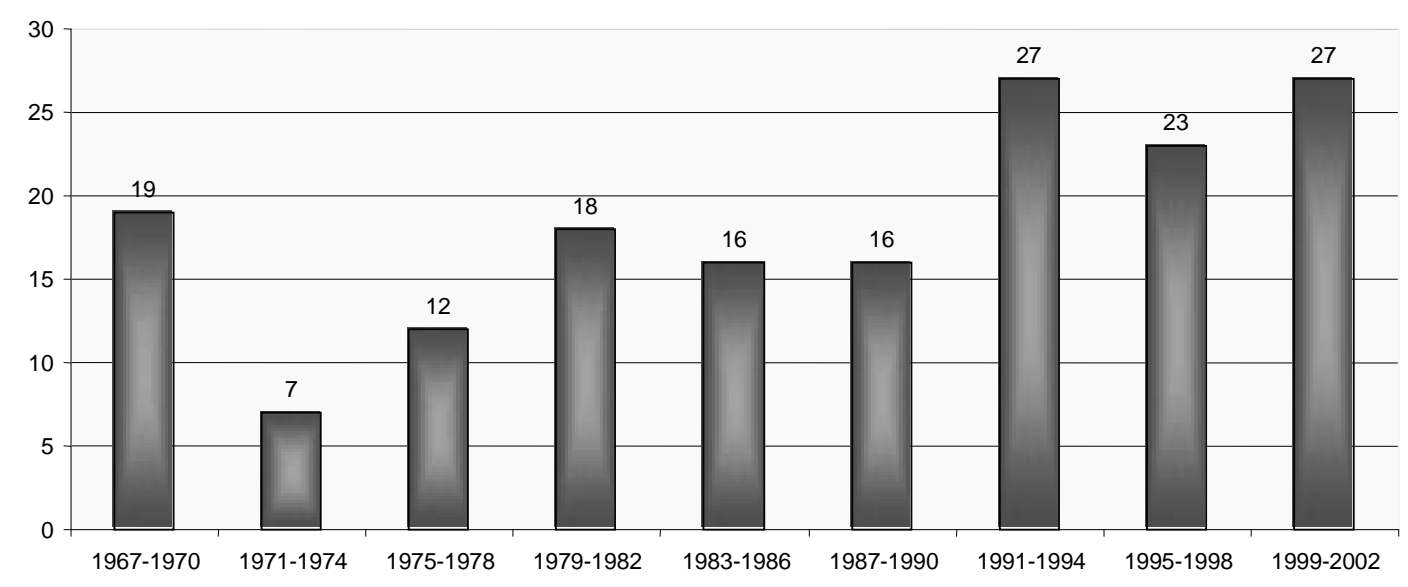

\section{Gráfico 1 - Distribuição da freqüência dos artigos sobre Ensino em Enfermagem por período (em anos). (São Paulo, 2003)}

As publicações encontradas sobre o ensino de enfermagem foram classificadas em cinco categorias distintas. A Tabela 1 mostra a classificação das categorias dos artigos e sua respectiva freqüência no período estudado.

Tabela 1 - Distribuição das categorias de artigos sobre ensino de enfermagem publicados entre 1967 a 2002. (São Paulo, 2003)

\begin{tabular}{lcc}
\hline \multicolumn{1}{c}{ Categoria do artigo } & Freqüência & Porcentagem (\%) \\
\hline Pesquisa & 78 & 47,3 \\
Revisão científica da literatura & 61 & 37,0 \\
Nota e discussão & 5 & 3,0 \\
Resumo de teses e dissertações & 5 & 3,0 \\
Relato de experiência profissional & 16 & 9,7 \\
\hline Total & 165 & 100 \\
\hline
\end{tabular}

No Gráfico 2 encontra-se a distribuição das categorias de artigos relacionando-os com o período de 4 em 4 anos. Verifica-se a oscilação da freqüência das categorias de artigos no decorrer dos anos, sendo observado uma importante inversão entre a freqüência dos artigos da categoria revisão crítica da literatura e da categoria pesquisa. Esta inversão é verificada na década de 80 , um reflexo do currículo mínimo aprovado em 1972, pelo Parecer 163/72 e Resolução 4/72, e que foram organizados após a promulgação da Lei de Reforma Universitária de 1968, que expressa a intenção de que o curso de graduação deveria desenvolver potencial tecnológico, científico, político e social nos alunos ${ }^{(8)}$. 


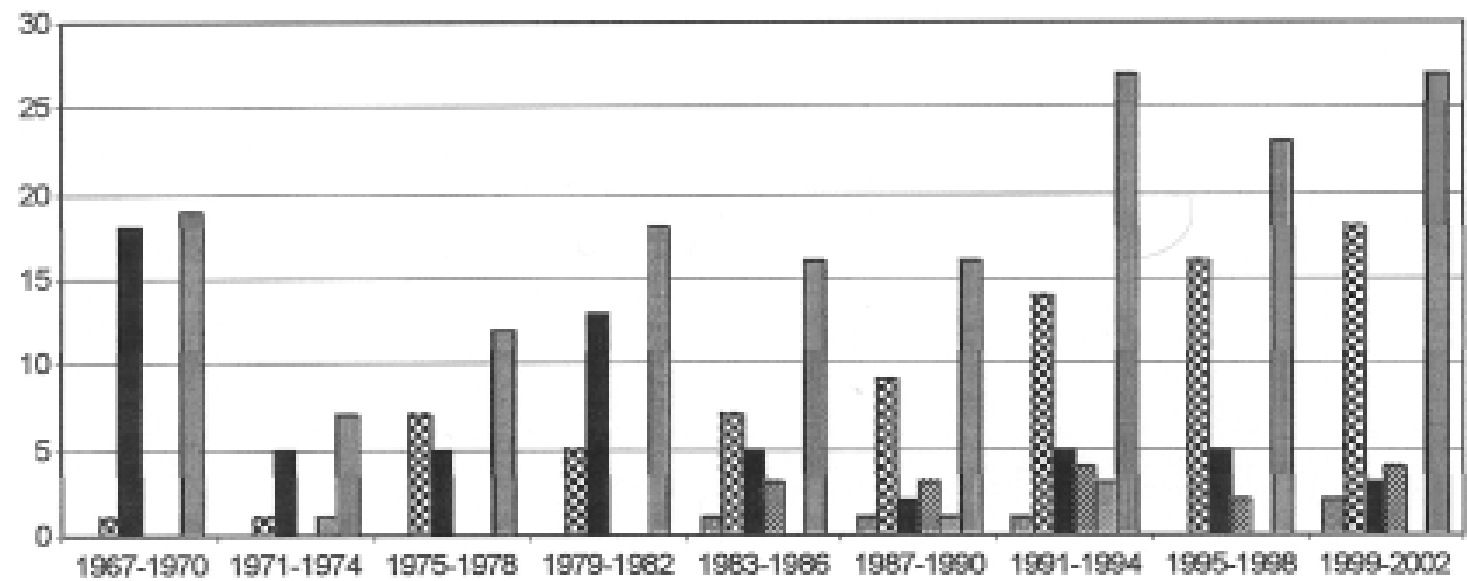

\begin{tabular}{|c|c|c|}
\hline ENata e discussรืం & a Pesquisa & - revisabo ciertifica da literatura \\
\hline 용 Relato de experiência & 口 Resumo tese ídissertaçalo & EGrand Total \\
\hline
\end{tabular}

\section{Gráfico 2 - Distribuição da freqüência das categorias de artigos publicados por período. (São Paulo, 2003)}

A Tabela 2 demonstra a distribuição das publicações segundo a classificação da ocupação do autor principal. Verifica-se uma expressiva freqüência $(85,5 \%)$ de autores docentes de enfermagem. Este dado demonstra que as produções científicas ficam concentradas no meio acadêmico realizadas por docentes, sendo que somente a partir da década de 70 há participação de alunos de pós-graduação. Nesta época, o ensino de enfermagem apresentou importante progresso com a criação de cursos de pós-graduação e investimentos na pesquisa. Em 2001, houve a primeira publicação de artigo, referente ao ensino de enfermagem realizado por enfermeiros assistenciais, podendo ser um reflexo do distanciamento entre o enfermeiro assistencial e o ensino de enfermagem, apesar deste ter importante participação no processo de ensino - aprendizagem do aluno durante os estágios.

Tabela 2 - Distribuição das publicações segundo a classificação do autor principal. (São Paulo, 2003)

\begin{tabular}{lcc}
\hline \multicolumn{1}{c}{ Autor principal } & F reqüência & Porcentagem (\%) \\
\hline Aluno de graduação & 8 & 4,8 \\
Aluno de Pós-Graduação & 11 & 6,6 \\
Docente de Enfermagem & 141 & 85,5 \\
Enfermeiro Assistencial & 2 & 1,2 \\
Sem Classificação & 3 & 1,9 \\
\hline Total & 165 & 100
\end{tabular}

As publicações de artigos, em sua grande maioria, estão direcionadas ao ensino de enfermagem no nível de graduação $(89,0 \%)$, sendo que $7,3 \%$ e $3,7 \%$ dos artigos se referem à pós-graduação e ao ensino médio respectivamente. Há significati- va concentração de pesquisas relacionadas ao ensino de graduação e os outros níveis são menos explorados apesar do número expressivo de trabalhadores de nível médio de profissionais na área de enfermagem (Tabela 3 ).

Tabela 3 - Distribuição das publicações sobre ensino de enfer-magem segundo o nível de ensino. (São Paulo, 2003)

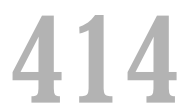

Rev Esc Enferm USP 2005; 39(4):409-16.

\begin{tabular}{|c|c|c|}
\hline Nível de ensino & F reqüência & Porcentagem (\%) \\
\hline Graduação & 147 & 89,0 \\
\hline Pós-Graduação & 12 & 7,3 \\
\hline Médio & 6 & 3,7 \\
\hline Total & 165 & 100 \\
\hline
\end{tabular}


Em relação à temática das publicações (Tabela 4), foram consideradas todos os níveis de ensino. Podemos destacar o expressivo interesse dos pesquisadores em relação ao conteúdo das disciplinas de enfermagem $(37,5 \%)$, o que denota a importância e preocupação com a formação do profissional de enfermagem, seguidos da temática sobre os alunos de enfermagem (19,3\%). O interesse pelos currículos de enfermagem concentrou-se no final da década de 60, onde foram significativas as discussões sobre o currículo de enfermagem no Brasil, época da Reforma Universitária ${ }^{(3)}$.

\section{Tabela 4 - Distribuição dos artigos sobre ensino de enfer- magem segundo a temática das publicações. (São Paulo, 2003)}

\begin{tabular}{lcc}
\hline Temática dos artigos & F reqüência & Porcentagem (\%) \\
\hline Conteúdo de disciplinas & 62 & 37,5 \\
Aluno & 32 & 19,3 \\
Estratégias de ensino & 23 & 14,0 \\
Estágio & 14 & 8,5 \\
Currículo & 13 & 7,9 \\
Docente & 10 & 6,1 \\
Avaliação & 9 & 5,5 \\
Vestibular & 2 & 1,2 \\
\hline Total & 165 & 100 \\
\hline
\end{tabular}

\section{CONCLUSÕES}

Acreditando que a abordagem do ensino de enfermagem seja um aspecto essencial para o desenvolvimento da profissão, é necessário o desenvolvimento de produções científicas na área.

Nos 36 anos pesquisados, identificou-se oscilação na frequiência de publicações sobre o tema, sendo observado um aumento considerável nos últimos 12 anos quando, historicamente, observamos reformulações importantes no ensino superior e no próprio ensino de enfermagem.

Em relação a categoria do artigo publicado, é importante ressaltar que no início, as publicações do tipo revisão científica da literatura se destacavam em relação aos demais e, a partir de 1983, este dado foi se modificando gradativamente, ocorrendo a inversão da frequiência entre artigo deste tipo e do tipo pesquisa; atualmente é mais freqüente os artigos do tipo pesquisa. Este dado caracteriza a preocupação dos enfermeiros em realizar pesquisas.

$\mathrm{O}$ aumento das publicações de artigos do tipo pesquisa na década de 90 , vem ao encontro das exigências do novo currículo (Parecer 314/94) ${ }^{(15)}$ que previu a formação do enfermeiro em quatro áreas: assistência, gerência, ensino e pesquisa; o discente, ao término de seu curso, deve apresentar uma pesquisa de conclusão da graduação, favorecendo a produção científica.

Constata-se um importante aumento do número de publicações de artigos, independente de seu tipo, realizada por docentes, alunos de pós-graduação ou alunos de graduação com evidente concentração no meio acadêmico.

As produções científicas fornecem importantes contribuições para o exercício da enfermagem, sendo assim, é de suma necessidade que os enfermeiros assistenciais realizem juntamente com os docentes de enfermagem, trabalhos científicos relacionados ao ensino de enfermagem, uma vez que, são profissionais que contribuem na formação do aluno de enfermagem, independente do nível de ensino, principalmente no que diz respeito aos estágios, favorecendo assim aintegração entre a teoria e a prática.

Na presente pesquisa, as publicações encontradas foram classificadas de acordo com o nível de ensino, constatando-se que $89 \%$ das pesquisas são referentes ao nível superior de enfermagem. Esta característica demonstra uma preocupação com o ensino do graduando de enfermagem, sendo este o principal profissional, agente de transformação e responsável pelo desenvolvimento da enfermagem, porém é importante ressaltar a necessidade de despertar o interesse em desenvolver pesquisas científicas na área do ensino médio profissionalizante, uma vez que a força de trabalho na enfermagem é constituída principalmente por estes profissionais.

A flexibilidade contida na nova LDB (Lei n ${ }^{\circ} 9394$ de 20 de Dezembro de 1996) ${ }^{(16)}$, dá às instituições formadoras a liberdade de determinar o perfil de aluno que deseja formar. No estudo realizado percebese uma preocupação com o conteúdo das disciplinas na formação dos futuros enfermeiros, o perfil do graduando de enfermagem, as estratégias de en- 
Elaine Emi lto

Regina Toshie Takahashi sino e as abordagens sobre o currículo desenvolvido pelas escolas.

Nesta perspectiva, as pesquisas referentes ao ensino de enfermagem devem corresponder às necessidades e tendências do momento influenciadas pela prática da enfermagem e com a preocupação na qualificação da formação destes profissionais.
A análise descritiva dos artigos publicados referente ao ensino de enfermagem desperta a preocupação sobre o desenvolvimento de pesquisas relacionadas ao tema e o acompanhamento das mudanças nas legislações e exigências do mercado de trabalho.

\section{REFERÊNCIAS}

(1) Fuszard B. Innovative teaching strategies in nursing. Rockville: Aspen; 1989.

(2) Germano RM. Educação e ideologia da enfermagem no Brasil. São Paulo: Cortez; 1985.

(3) Oguisso TA. A legislação do ensino de graduação em enfermagem. Rev Esc Enferm USP 1976; 10(2):202-18.

(4) Dilly CM. Processo educativo em enfermagem: das concepções pedagógicas à prática profissional. São Paulo: Robe; 1995.

(5) Nimtz MA. O ensino da disciplina de administração em enfermagem nas escolas de graduação da grande São Paulo. [dissertação] São Paulo (SP): Escola de Enfermagem da USP; 1999.

(6) Franqueiro JNV. Formação gerontológica em um curso de graduação em enfermagem: análise curricular mediante as novas diretrizes da educação. [dissertação] Ribeirão Preto (SP): Escola de Enfermagem de Ribeirão Preto/USP; 2002.

(7) Nimtz MA. Ciampone MHT. O ensino da disciplina de administração em enfermagem nas escolas de graduação da grande São Paulo. Rev Paul Enferm 2001; 20(1):22-30.

(8) Rodrigues RM. Teoria e prática assistencial na enfermagem: o ensino e o mercado de trabalho. [dissertação] Ribeirão Preto (SP): Escola de Enfermagem de Ribeirão Preto/USP; 2000.

(9) Galleguillos TGB, Oliveira MAC. A gênese do desenvolvimento histórico do ensino de enfermagem no Brasil. Rev Esc Enferm USP 2001; 35(1):80-7.
(10) Conselho Nacional de Educação. Câmara de Educação Superior. Resolução CNE/CES n. 3, de 7 de setembro de 2001. Institui diretrizes curriculares nacionais do curso de graduação em enfermagem. Diário Oficial da República Federativa do Brasil, Brasília, 9 nov. 2001. Seção 1, p. 37.

(11) Tronchin DMR. O enfermeiro assistencial e a formação do aluno de graduação. In: Anais do $4^{\circ}$ Encontro de Enfermeiros de Hospitais de Ensino do Estado de São Paulo; 1997 jul. 31- ago. 02; São Paulo. São Paulo: Hospital Universitário da USP; 1998. p. 209-21.

(12) Ewan CE. Teaching nursing. A self instructional handbook. New York: Croom Helm; 1984

(12) Cervo AL. Metodologia científica. São Paulo: Prentice Hall; 2002.

(14) Stefanelli MC, Carvalho DV, Elsas BX, Tsunechiro MA, Posso MBS, Lui MC. Análise da Revista da Escola de Enfermagem da USP: período de 1967 a 1981. Rev Esc Enferm USP 1987; 21(3):263-84

(15) Ministério da Educação. Conselho Federal de Educação. Parecer 314, de 06 de abril de 1994. Diretrizes do currículo mínimo para o curso de enfermagem. Brasília (DF); 1994.

(16) Brasil. Lei n. 9394, de 20 de dezembro de 1996. Estabelece as diretrizes e bases da educação nacional. Lei de Diretrizes e Bases da Educação - LDB. [on line] Brasília, 1996. Disponível em: <http:// prolei.cibec.inesp.gov.br> (14 fev. 2002)
Correspondência:

Elaine Emi Ito

Rua Brígida de

Vasconcelos, 62

CEP -08290-650 - SP

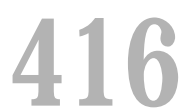

Rev Esc Enferm USP 2005; 39(4):409-16. 